\title{
Dispositivos para la transformación de la subjetividad en la psicoterapia sistémica
}

\section{La psicoterapia como un modo de relación}

1 ás que la definición de lo que es la psicoterapia, lo interesante ción. Porque la psicoterapia es una interacción. Es un encuentro entre humanos y por tanto se fundamenta en la estructuración de una relación a partir del momento en que se inicia el proceso, dentro de los llamados contexto y sistema terapéuticos.

No necesariamente todo encuentro psicoterapéutico toma un largo tiempo. Puede haber encuentros únicos con efectos terapéuticos, porque se dan procesos que movilizan el cambio, dado que lo terapéutico consiste en activar las posibilidades de resolución, de mejoría y de transformación, gracias a que aparecen otras perspectivas y se descubren dimensiones de la vida antes ignoradas. Por lo tanto se diría que la psicoterapia es una experiencia de encuentro humano, de la cual pueden emerger cambios inmediatos, entendiendo que se trata de una conversación con sus propias características.

Surge así la pregunta por ¿cuáles son los dispositivos que favorecen el cambio y con qué de las personas se trabaja? ¿Qué es lo que 
entra en juego en la psicoterapia precisamente para que su efecto sea terapéutico? Hablar de "psico"-terapia implica entrar en los mundos de la subjetividad y la intersubjetividad que participan en la conversación. En ese sentido se diría que el cambio se da intencionalmente sobre procesos psicológicos, los cuales incluyen todas las vivencias y capacidades humanas, organizadas para efectos de comprensión en procesos cognitivos, afectivos e interaccionales, que circulan de forma más o menos consciente y que se evidencian en la verbalización suscitada en la conversación. Esos procesos son autopoiéticos, porque simultáneamente se construyen y configuran los sistemas de relación inherentes a la vida de cada persona; es decir, la percepción, la emoción, la memoria y los demás procesos adaptativos se activan en la interacción con los demás y con el mundo.

Se trata entonces en la psicoterapia con un sujeto que tiene un mundo, valga la redundancia, subjetivo, interno y propio, que emerge y se transforma permanentemente en las relaciones con los demás en los distintos ámbitos de relación. Los procesos psicológicos permiten dicha emergencia de un sujeto, de un ser que se diferencia de otros, paradójicamente en constante relación con los demás en sus contextos significativos.

En el encuentro terapéutico ese ser se pone en marcha, emerge a partir del modo como se conversa sobre las situaciones vividas, donde ahora, mediante el relato suscitado en la conversación, el terapeuta es incluido y participa como ser en el mundo, en la representación y en la recreación de la situación. La conversación terapéutica es eficaz si favorece esas nuevas versiones y visiones y ello exige la participación del terapeuta con su propio mundo de vivencias. Si esto no ocurre, solo observará conductas cuyo sentido tendrá poco que ver con el contexto del consultante y con el encuentro en sí y se remitirá a las teorías o a otros referentes que, al no hacerse conscientes, operan como automatismos que reproducen prejuicios, juicios morales o simples convencionalismos culturales que más bien pueden afianzar las pautas que mantienen el sufrimiento motivo de consulta.

Porque si bien la conversación terapéutica tiene que tener un propósito cuyo cumplimiento habla de la eficacia terapéutica, hay partes 
de ella que son acordadas y otras que son inciertas, porque justamente su eficacia implica novedad, para que emerjan vivencias y apreciaciones que no estaban pensadas, pero que coadyuvan al propósito consensuado acerca de la dirección y la finalidad de la conversación. Es en ese proceso donde participa el self en permanente movilización hacia el logro de lo que desea y que no ha podido alcanzar por sí mismo, razón por la cual acude a la psicoterapia.

Como lo plantea el antropólogo Michael Houseman (2003), la psicoterapia es un proceso paradójico en el que una o unas personas acuden a un tercero que es su terapeuta y le entregan una cuota de poder para que les ayude a lograr lo que quieren pero no han podido alcanzar por sí mismas. Es una paradoja en la que a veces se enredan los psicoterapeutas, intentando eludir el inevitable papel asimétrico que tienen en la relación de ayuda, bajo la fantasía de que no ejercen influencia. La paradoja consiste precisamente en que el terapeuta no puede ayudar a la persona a lograr lo que él cree que le conviene, sino en ayudarle a que ella logre lo que ella quiere y cree que le conviene. Por eso la tarea del terapeuta es favorecer la movilización de aquellas condiciones de la persona, pareja o familia que la conducirán a donde quiere estar para sentirse satisfecha.

Cuando las personas llegan a terapia tienen un self que esta enredado, estancado, frente a un panorama estrecho y hay que ayudarles a que lo amplíen, lo liberen y lo desatasquen. Su self está confundido, perplejo, arrinconado, callado y tiene detenida su capacidad natural de adaptación con autonomía. Por lo tanto el propósito de la terapia es crear un contexto de relación que viabilice ese proceso, para lo cual el terapeuta tiene que estar dispuesto a comprometerse en la gestación de ese contexto de relación. El compromiso incluye la capacidad de resonancia con el ser humano que es el consultante y la creación de una relación de confianza donde pueda exponer sin riesgos su self, su mundo interior, su mundo de vida. No se trata de un intercambio de información sino del desarrollo de una conversación protegida, donde el consultante se aproxima a su sufrimiento contando con la consideración humana del terapeuta. Porque aquí confianza representa sobre todo confidencialidad y ser acogido sin juzgamientos ni condicionamientos. 
No obstante, la psicoterapia implica también un contrato, el cual a su vez es una condición de construcción de la confianza y de la distinción entre una conversación cotidiana y una conversación psicoterapéutica. Hay un contrato económico, hay unas condiciones contextuales temporo-espaciales que se precisan en la llamada definición del contexto, para que ese ambiente quede libre de confusiones que empañen la relación de ayuda.

La transparencia sobre lo que se va a hacer, cómo, cuándo, dónde, es uno de los ingredientes para generar confianza. También lo es la transparencia del terapeuta sobre su manera de pensar acerca de la terapia, su manera de trabajar, en cómo concibe su trabajo y aquellos aspectos de la vida que tocan con el sufrimiento del consultante. En este sentido, la psicoterapia sistémica se distingue de otras porque más que una terapia de escucha es una terapia de diálogo, de conversación, donde el terapeuta expresa sus apreciaciones y sus puntos de vista pertinentes para expandir la experiencia del consultante. Es decir, es un encuentro que avanza a través de la interacción y no simplemente de la interpretación. Esta es una terapia donde consultante y terapeuta son actores activos en una relación horizontal con roles diferenciados coherentes con el contexto de ayuda.

Esto lleva también a diferenciar los dispositivos del cambio. En general, las terapias de escucha parten de la premisa de que la catarsis o la descarga emocional es terapéutica en sí misma y por eso se propicia, dejando que, supuestamente, fluya el sujeto libremente; no obstante, desde un punto de vista contextual y comunicacional, se sabe que el sujeto no está libre, pues la presencia y la manera de ser de esa presencia del otro, actúan como señales que le indican de qué puede hablar, de qué no y cómo. Por otra parte, una visión simplista de la descarga emocional como dispositivo de cambio implica una idea del psiquismo como una vasija pasiva que se llena de algo muy desagradable que hay que vaciar; por supuesto no se niega el alivio que acompaña a la catarsis, pero también se piensa que esta descarga sería tan solo un mecanismo homeostatizante que puede favorecer la cronificación de los problemas.

Por el contrario, la psicoterapia conversacional es de carácter reflexivo, donde las interpretaciones no se basan en conceptos sino en 
la experiencia vivida y encarnada por el sujeto a través de su historia; la reflexión pretende hacer distinciones y proponer la construcción de mundos de vida diferentes que conduzcan a ciertas rupturas que marquen el cambio de vida, a partir de la apropiación de la propia experiencia. En la interpretación, quien la hace generalmente acude a un marco de referencia teórico, ajeno a la experiencia del otro y por eso genera un proceso cognitivo que en muchos casos lleva a que dicha experiencia encaje dentro de ese referente que le es prestado y superpuesto a su experiencia, aunque sea validado por la autoridad profesional y académica de quien conduce la terapia.

En consecuencia, ese tipo de terapias corre el riesgo de condicionar al ser humano a un discurso que encarna la connotación de fuente de poder, de verdad y de control, que iría en contravía de los procesos reflexivos cuya dirección es el ejercicio de la libertad, la búsqueda de la expansión del espíritu humano y de la reconstrucción de la vida sobre la base de una nueva propuesta que surge en el encuentro.

En cuanto la psicoterapia es un encuentro contextual, los psicoterapeutas aprenden a estar dispuestos a entrar en el contexto del consultante, para que desde allí faciliten la activación del cambio de significaciones y de comportamientos en sus interacciones vitales. Es decir, desde allí los procesos reflexivos con los consultantes parten de sus propias teorías de la vida, de sus propias vivencias y sus propios deseos, pues el cambio se inicia cuando, dueños de su experiencia, la resignifican y rediseñan su curso futuro, sin tomar prestado de un tercero lo que debería ser su vida. En ese sentido, la participación del terapeuta consiste en conducir el proceso de cuestionamiento y de reflexión para que el consultante reorganice su marco de referencia y su historia narrada y encarnada, lo cual lleva inevitablemente a hacer ajustes a la propia identidad.

\section{Papel de las teorías psicológicas de la psicoterapia}

Se puede decir que la teoría es el referente metafórico para apoyar el proceso terapéutico, pero nada más. Como metáfora, una teoría puede 
decir por ejemplo que ciertas relaciones están inconclusas o confusas, pero el verdadero sentido de su inadecuación radica en la historia misma del caso. Juntando las teorías psicológicas con las de los consultantes surge una nueva teoría, y si se juntan varios casos pues surgirá una teoría más general acerca de la vida. Por eso, es posible pensar la psicoterapia como un espacio para construir nuevas teorías, nuevas maneras de explicar y de ver la vida con cada consultante. Esto exige gran flexibilidad del terapeuta, para poder aceptar las distintas versiones y validarlas contextualmente, sobre todo con una mirada de verosimilitud y de probabilidad.

El hecho de que un terapeuta conozca las teorías que explican diversos fenómenos humanos tendría también el valor de ampliar su propio marco de referencia, para que su esquema de la vida no se le reduzca al de sus propias experiencias, sino que pueda, como lo dice Salvador Minuchin (1981), llevar en su morral muy variadas herramientas, dado que, a pesar de que tengamos elementos comunes por ser miembros de la especie, en el arte de la vida cada quien pinta el cuadro a su manera. Además, autonomía y libertad están asociadas a variedad y a diversidad y como terapeutas es necesario poder jugar con muchas metáforas, para ser pertinentes y respetuosos en cada caso.

Es en esa oportunidad de variedad donde el psicoterapeuta también avanza en su desarrollo personal en cada sesión, en la medida en que tiene ocasión de mirar otros mundos y posibilidades. Así, la riqueza de sus metáforas será proporcional a la riqueza y a la versatilidad de su vida.

\section{La psicoterapia como espacio epistemológico}

Sin duda se construye conocimiento específico en la psicoterapia. Como se ha dicho, no se hace a partir del entrecruzamiento de teorías científicas, sino de múltiples historias, mitos, creencias y marcos de referencia que se entremezclan de una forma compleja y azarosa, porque son azarosas y caóticas las circunstancias en las que se construye la vida. Esto exige que el terapeuta acuda no tanto a la lógica aristotélica sino 
a todas las para-lógicas, con una disposición que se sale de catálogos y de protocolos y más bien se acoge a la intuición y la curiosidad para que el encuentro favorezca la novedad. Esto no significa que ante la ausencia de protocolos, el proceso psicoterapéutico avance sin rumbo y en forma aventurada, pues el referente constante es la orientación del cambio planteada por el consultante, así ella misma sea cambiante en el tiempo.

Adicionalmente, como se ha venido diciendo, el proceso terapéutico se rige por los principios de reflexividad, contextualidad y autorreferencia, los cuales en cuanto tienen que ver con procesos cognitivos se apartan de la perspectiva representacional. Esto tiene importantes implicaciones en cuanto a los dispositivos del cambio, porque no se trata de modificar las representaciones archivadas sino por el contrario de poner en el juego de la conversación reflexiva esas representaciones, para adscribirles nuevos sentidos desde su condición como experiencia encarnada en la vivencia, en la comunicación y en la acción.

\section{Psicoterapia con individuos y con sistemas significativos de relación}

La psicoterapia con los sistemas significativos permite ubicar el self en el mundo interaccional, pues es en el contexto de las relaciones donde se da su recomposición. No obstante, esto no exige la presencia física de todos los familiares, pues con una persona sola se pueden evocar sus distintos sistemas de referencia para contextualizar su ser en esas relaciones. También en este sentido hay una diferencia con otras terapias que pretenden modificar las representaciones enfocando el cambio desde una óptica cognitiva racionalista, mientras aquí la cognición se considera como un fenómeno adaptativo, puesto en escena en el mundo de las relaciones.

La cognición se asume en la psicoterapia sistémica como una condición fenomenológica, no estructurada, lo cual anticipa que, evidentemente, se generan cambios en la conciencia individual y en las interacciones humanas, aprovechando lo que ya demuestran las 
investigaciones neuropsicológicas alrededor de la globalidad del cerebro y de su funcionamiento en red; esta visión de articulación permite que la psicoterapia tome en promedio cuatro a diez sesiones, porque si se trabaja con la fenomenología de las experiencias humanas el cerebro desarrolla todo su potencial de conectividad.

Esto permite remarcar que el proceso de la conversación terapéutica es un proceso vivencial, no racional, que moviliza toda la red cognitiva y así se confrontan y se trascienden las dicotomías que en la psicología separan razón, emoción, intuición, condición racional, pensamiento y sensación, las cuales se encadenan en la vivencia individual y en lo que podría llamarse la "convivencia del acto psicoterapéutico", la cual, en cuanto novedad para el sujeto, tiene un carácter de renovación de la experiencia.

Se diría que esa posibilidad de convivencia tiene existencia en un proceso reflexivo donde los consultantes y los terapeutas, como sujetos activos, salen renovados y sensibilizados para experimentar las circunstancias agradables y dolorosas y sentir placer, inquietud y serenidad.

En las prácticas muy concretas que se desarrollan en la conversación terapéutica, esto implica que también, a diferencia de otros enfoques, la psicoterapia sistémica no se centra en la historia de los hechos ni tampoco en su cronología, buscando en eventos específicos las explicaciones causales, dentro de una perspectiva de objetividad, como si la historia personal fuera un conjunto de datos de la "vida real". En la psicoterapia sistémica, la historia se basa en las experiencias vividas y narradas en este encuentro, las cuales en la conversación terapéutica van adquiriendo nuevos matices y significados.

Porque en la conversación terapéutica la experiencia vivida previamente se revive desde una postura reflexiva que permite activar esta capacidad de aproximación y de distanciamiento de la experiencia, de modo que fluidamente se pasa de sentirla a observarla desde afuera, para reevaluarla y reinterpretarla. En este sentido, la historia narrada es un referente metafórico que hace que los acontecimientos sigan una organización a través de un hilo temporal, cuyas conexiones son de hecho una creación del sujeto, y por eso es que se puede hablar de una reorganización de la historia personal y familiar. Esa reorganización 
empieza al desmenuzar el relato mismo del consultante, de modo que en esa forma se establecen las conexiones entre el relato de la experiencia vivida en el pasado y las que se viven en el presente, para poder actualizar la experiencia, y por decirlo así, reconfigurarla según las necesidades y posibilidades del consultante. Para ahondar en estos procesos es indispensable la existencia de la ya mencionada relación de confianza en el sistema terapéutico, entendiendo que solo así se genera el clima necesario para profundizar en los sistemas de significación, conectados siempre a los sistemas de vinculación.

De lo contrario, escuchar las historias de los consultantes, sin actualizarlas en la vivencia para transformarlas, puede conducir más bien a la consolidación de los llamados relatos dominantes que obstaculizan el bienestar y la evolución personal, pues se refuerza la vieja historia de sufrimiento o de malestar que ha hecho que la persona acuda a terapia y por lo tanto el proceso de narrar deja de ser una herramienta de cambio. Por eso hay que ir escuchando breves relatos, para poderlos ubicar en el presente, conectarlos y valorar la legitimidad, la validez y la vigencia que eso tenga para su vida.

Así se están también desplegando los principios epistemológicos inherentes a la terapia, es decir, que de la realidad de la cual se habla en la terapia es esa realidad construida interaccionalmente, sin cuyo reconocimiento sería imposible movilizar el cambio en los sistemas conyugales, familiares y grupales en general, dado que si se cree que la realidad de cada uno es "objetiva", y cada uno tiene una diferente, solo quedarían las negociaciones racionalistas donde todos ceden algo pero no necesariamente cambian sus sistemas de significación ni de vinculación y por lo tanto son acuerdos que resultan ser transitorios y poco potentes. En otras palabras, lo que interesa es que miren las distintas versiones para construir una nueva, donde quepa la humanidad de cada uno y así como sistema, reformulen colectivamente sus nuevas relaciones. Así se respetan también los principios de la diversidad, diferencia y distinción, vistos precisamente como la base del potencial del cambio del sistema.

Esto permite subrayar que, finalmente, lo único que podemos tener de la propia vida y de la de los demás son versiones. Por tanto, en 
la terapia no se trabaja con verdades objetivas, sino con verdades psicológicas, que pueden ser revisadas, cuestionadas y reconstruidas, las cuales reposan en los cuerpos y en la memoria vivencial, más allá de la lógica, como ya se ha dicho. Es esa ruptura con la lógica racional y la validación de lo diverso, lo que da paso a la novedad que conduce a movilizar los sistemas atascados.

Todo lo anterior lleva a remarcar que, en esta perspectiva se asume el desafío de abrir la "caja negra", y se considera que lo psicológico se encarna en el sujeto, la conciencia y el mundo subjetivo y no solo en la conducta ni en las profundidades inconscientes. Naturalmente no se trata de una conciencia racional sino de una conciencia experiencial, corporizada en el sistema de las relaciones, dentro de un orden de sentido. Se trata de una conciencia y de un sujeto ecológico en evolución, que surge en la compleja interacción de lo social, lo histórico, lo político, lo cultural y que constituye por tanto una dimensión bien diferenciada de la vida.

En ese proceso participan simultáneamente la emoción, la intuición y la memoria, de modo que se acude al acontecimiento pero para conectarlo con la experiencia. En ese proceso participan el sujeto pleno y la conciencia abierta a las posibilidades cambiantes que ofrece el movimiento flotante de la vida, donde se mueven las situaciones, las personas y los mundos de vida, los cuales así se reactivan y se logra salir de los estados de malestar y de estancamiento.

\section{La experiencia personal del terapeuta como lugar de resonancia}

La experiencia personal asociada por Mony Elkaïm (2004) con su noción de "resonancia", está ligada a la historia del terapeuta y solo se activa por la función que cumple en el contexto presente. La resonancia es la amplificación de elementos similares y comunes a los diferentes sistemas en interacción, la cual emerge en la intersección posible entre sus respectivas estructuras o construcciones del mundo. El ensamblaje ocurre cuando las resonancias están compuestas por elementos 
disímiles que pueden llegar a ser complementarios a las dificultades de los consultantes o interferentes con estas. Según Elkaïm (1989) son esas resonancias las que hacen posible el cambio en terapia. Ambos fenómenos se refieren a las intersecciones que se producen entre el mapa de mundo del consultante y el mapa de mundo del terapeuta. Gracias a este concepto, la paradoja de la autorreferencia deja de ser una limitación para convertirse en una posibilidad, si el terapeuta ha aprendido a utilizarse como instrumento terapéutico, utilizando la función sistémica de la resonancia para generar nuevas posibilidades para el sistema terapéutico.

Siempre estamos en resonancia, pero en general, la necesidad de analizarla aparece cuando la brecha entre la historia del terapeuta y la de los consultantes afecta la flexibilidad del sistema en función del cambio, como cuando la interacción de las construcciones del mundo del terapeuta y el consultante producen una danza tan repetitiva que la persona no puede avanzar.

Ese análisis debe adaptarse a contextos específicos. Por ejemplo cuando el supervisor trabaja con un equipo terapéutico, no se recomienda exponer a los miembros a hablar de su historia personal ante sus colegas. Puede más bien ser útil el uso de metáforas: se puede pedir al terapeuta contar una historia, describir lo que ve por la ventana, o incluso contar un cuento de hadas conocido y esto va realmente a adaptarse y a relatarse de una manera reveladora. Estas herramientas metafóricas son muy útiles en situaciones donde uno invita al otro a hablar sin exponerse. El que habla entiende que sea de lo que se trate él vive de otro modo lo que los limita, mientras que el grupo circundante tiene una idea general sin necesidad de conocer su historia exacta.

El estudio de la resonancia permite entonces, dentro de este contexto donde sujeto y objeto son inseparables, pensar esta inseparabilidad que subsume el concepto de sistema terapéutico y ofrecer al terapeuta un riguroso contexto de hipotetización y de intervención.

Esta aproximación, enmarcada en la cibernética de segundo orden, que pone el acento en el vínculo que une al observador con el sistema observado trae consigo un problema importante: ¿cómo el terapeuta 
puede entonces, si no es separable del sistema sobre el cual formula hipótesis, verificarlas? ¿Cómo puede tener un discurso "válido” y cómo puede intervenir?

Como dice Elkaïm (2004), una primera condición para resolver dichos dilemas es que el terapeuta acepte que sus hipótesis son inseparables de sí mismo, de su historia y de aquello que la constituye. Debe también conservar la idea de que ellas surgen en un contexto dado donde hay más personas en juego.

Por ejemplo, si la experiencia de cada miembro de la pareja está por supuesto relacionada con su historia personal, también hay otros factores sin cuya presencia tales elementos no se habrían activado. Un elemento se amplifica cuando, en circunstancias especiales, el enlace con otro lo despierta. Así surge una consecuencia bastante paradójica: lo que reprochamos a nuestro socio, con frecuencia tiene la función de fortalecer nuestra propia visión del mundo y evitar exponernos al descubrimiento reiterado que la realidad es de hecho como tememos que sea. La experiencia individual de cada persona también tiene una función en relación con el contexto sistémico en el que surge y se mantiene. Es decir, hay contextos donde será poco probable que se reviva una experiencia porque no tendría allí ninguna función. Se crea así una escena afectiva y cognitiva nueva que sería lo que la terapia puede evocar si el terapeuta no participa en la danza dolorosa a la que los consultantes lo pueden invitar. Esta no activación del elemento latente rompe la repetición y puede así amplificar la vivencia y aportar a la construcción de una nueva visión del mundo que trascienda la experiencia dolorosa. Vista así, no es posible aceptar la idea del pasado como una prisión; nuestra historia nos sensibiliza pero no nos domina.

En cuanto a la construcción de hipótesis con base en las evocaciones que las narraciones de los consultantes generan en el terapeuta, la pregunta fundamental es acerca de la función que tiene para el consultante la vivencia evocada por el terapeuta. En general, si el terapeuta no se metaposiciona con respecto a tal vivencia, su respuesta podrá activar la reiteración de la pauta relacional y reforzar las creencias profundas que han alimentado el malestar a lo largo de su vida. Esa metaposición debe conducir a que al no atraer solamente su propia historia, el 
terapeuta invite al consultante dentro de la dinámica contextual de la psicoterapia a ampliar los componentes de sus creencias profundas.

Por lo tanto, el terapeuta debe comprender su vivencia no solo con referencia a su propia historia sino también analizar la función presente de esa vivencia con respecto a los consultantes. Esto es lo que Elkaïm llama resonancia; esta configuración particular donde las personas pertenecientes a un mismo sistema "vibran" sobre el mismo tema. En este sentido, la resonancia no es empatía, contratransferencia, ni reacción en espejo; este concepto muestra mejor su validez heurística cuando se estudia la función de una resonancia dentro del sistema humano donde los miembros vibran, en función de reforzar las creencias profundas de este sistema.

Cada uno responde de una cierta manera porque es sensible a lo que el otro le trasmite. Si un comportamiento encuentra resonancia en la otra persona, ese comportamiento o esa pauta se va a amplificar. Así cada una de las reglas potenciales se puede amplificar si encuentran una resonancia, una intersección en el otro. Se puede decir que una regla o un tema se amplifican aún más cuando resuenan en diferentes personas pertenecientes a distintos sistemas, cuando esa regla o tema corresponde a un determinado nivel de funcionamiento de cada uno.

Por algo dos acepciones de resonancia son un eco que se produce en el espíritu y el aumento de amplitud de una oscilación bajo la influencia de impulsos periódicos de frecuencia cercana. Elkaïm (1989, 2004) denomina resonancia a los conjuntos particulares, constituidos por la intersección de elementos similares y comunes en diferentes individuos o diferentes sistemas humanos, con base en los cuales se suscitan las construcciones mutuas de lo real en el sistema terapéutico. Esos elementos resuenan bajo el efecto de un factor común, un poco como los cuerpos vibran bajo el efecto de una determinada frecuencia. Este fenómeno surge en forma impredecible y subjetiva y concreta zonas de interacción y de creencias que merecen ser explorados con rigor. Por eso es importante que el terapeuta se pregunte por los sentimientos que un consultante o una familia le despiertan, por su propia vivencia, por el sentido de tal vivencia en ese contexto específico y sobre todo por la utilidad de dichas vivencias para el consultante, 
de modo que se instaura un diálogo interior en el terapeuta, gracias al cual se enriquece el diálogo con el consultante. En ese diálogo interior se revela el mundo subjetivo del terapeuta.

Probablemente el consultante va a intentar modelar el comportamiento del terapeuta para repetir con él una pauta significativa, que si bien tiene una función adaptativa de protección, también mantiene el no cambio y surge como una forma de neutralizar la amenaza que representa la intrusión de este extraño a quien por lo tanto intentará introducir en su sistema. Es aquí donde entra en juego el accionar creativo del terapeuta para moverse dentro y fuera del mundo de los consultantes y para hacerles invitaciones fructíferas a que se arriesguen a ensayar nuevas formas de relación y a cuestionar sus creencias profundas.

\section{Resiliencia y resonancia}

En la resonancia y la resiliencia las emociones están siempre presentes. De hecho, si el trabajo a partir de resonancias sitúa al terapeuta en el campo de las emociones, el desarrollo de un contexto terapéutico que promueve la resiliencia también pertenece al mundo de las representaciones afectivas. Por lo tanto hay que tejer las intervenciones consultando el sentido de la narración para los consultantes y los terapeutas. En este nivel, el entretejido entre resonancia y resiliencia es posible y funciona tanto en la dirección de retroacciones negativas que se oponen al cambio y mantienen a los consultantes en una demanda imposible cuyo futuro será el sufrimiento y la resignación, como en la dirección del cambio, donde el terapeuta a partir de sus resonancias, se convierte en factor de resiliencia. Pero el límite entre estas dos alternativas es muy borroso.

El terapeuta no puede escapar a sus emociones. Bajo el ángulo de la resiliencia, se puede considerar que después de darle sentido a las representaciones vividas por el terapeuta, este puede crear un proceso tutor de desarrollo y ser él mismo un factor de resiliencia. Esto implica que el terapeuta escuche su cuerpo y su mente, identificando en el cuerpo signos de intensificación emocional como aumento del ritmo 
cardíaco, de la temperatura, titubeos, cambios en el tono de voz, repetición de actos o de frases estereotipadas, y pueda reconocer la naturaleza de la emoción subyacente, sea alguna forma de miedo, tristeza, rabia, o también entusiasmo y afecto. A veces puede ser necesario hacer pausas en las sesiones para digerir esas emociones e identificar sus conexiones con la historia personal del terapeuta.

Maestre (Cyrulnik, Elkaïm \& Maestre, 2010) se cuestiona por la diferencia entre resonancia y proyección y resuelve la duda siguiendo el criterio expuesto por Elkaïm $(1989,2004)$ según el cual, para verificar si hay resonancia lo que corresponde es que después de identificar el sentido que las emociones evocadas tienen para el terapeuta, él se plantee varias preguntas: ¿cómo este tema que le parece esencial al terapeuta es importante para los consultantes?, ¿cómo les concierne? Si las respuestas no le permiten elucidar cómo esos asuntos son relevantes para ellos, debe renunciar al menos temporalmente a insistir sobre el tema, pues de otro modo se expone a dos grandes dificultades: una, invadir al sistema consultante concentrándose en elementos que tienen sentido para él, pero que son muy secundarios para los miembros de la familia y la otra, provocar fuertes resistencias en ellos. Elkaïm (Cyrulnik, Elkaïm \& Maestre, 2010), por lo tanto insiste en que cuando en una relación de ayuda algo de lo que ocurre saca al terapeuta de su neutralidad benevolente, es importante que se pregunte no solo a qué circunstancias de su historia lo envía, sino cuál es la utilidad de esa experiencia para las creencias profundas del consultante.

\section{Los terapeutas como tutores del desarrollo}

En el mismo texto, Cyrulnik recuerda que la activación de la resiliencia incluye tres elementos:

1. La recuperación de los recursos internos del consultante, los cuales, desde su punto de vista están impregnados en el temperamento y en las interacciones tempranas pre verbales durante el primer año de vida; ellos además explican la forma de 
reaccionar a las agresiones de la vida y de aplicar más o menos exitosamente los que llama guardianes del desarrollo.

2. La estructura de la agresión, daño, lesión o falta, pero sobre todo el significado que le da más adelante la familia y el contexto social en la historia de quien la sufre. Ese significado explica los efectos devastadores del hecho que se convierte en trauma.

3. La posibilidad de contar con espacios de afecto, actividades y palabras de las que dispone a veces la sociedad, donde encuentran tutores de la resiliencia que le permitirán retomar el desarrollo afectado por la lesión.

Se diría entonces que uno de tales dispositivos sociales es la psicoterapia. En ese sentido, el contexto terapéutico es favorable cuando los consultantes fluyen en el abordaje de un tema relacionado con las emociones en resonancia y cuando las intervenciones del terapeuta aportan información en el entendido de Bateson, es decir, comunican algo que crea una diferencia. Es dentro de este tipo de procesos que Maestre (Cyrulnik, Elkaïm \& Maestre, 2010) considera que el terapeuta se convierte en un "tutor de la resiliencia" pues se genera un clima relacional con gran proximidad pero también con gran diferenciación, donde interactúan los elementos compartidos de la visión del mundo de todos los participantes. 